\title{
Modifications selon la LPTh: marché et sécurité
}

\author{
Carlos Beat Quinto
}

Dr méd., membre du Comité central de la FMH, responsable du département Santé publique, professions de la santé et produits thérapeutiques

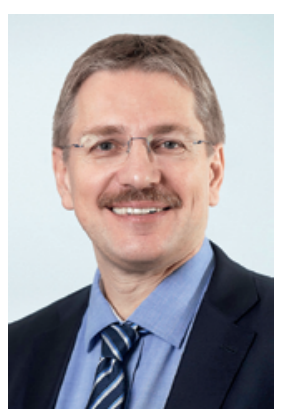

Pour la prescription et la remise de médicaments, notre cabinet de groupe (médecine de famille) applique la devise: le moins possible, mais autant que nécessaire. Pour ma part, depuis que j'ai travaillé au siège d'une société agissant dans le domaine de la sécurité des médicaments, je crois aussi à cette maxime: «There are no safe drugs, there are only safe doctors.» Alors que la libéralisation de la publicité pour les médicaments a déjà dopé la demande de consultations dans les cabinets médicaux et les pharmacies, des modifications supplémentaires sont encore prévues.

Notre cabinet propose à chaque patient la solution qu'il souhaite, à savoir la remise directe de médicaments par le médecin ou une ordonnance pour qu'il puisse se rendre à la pharmacie de son choix. D'après la nouvelle loi sur les produits thérapeutiques, une ordonnance devrait être délivrée à chaque fois, même si le médicament est remis directement par le médecin traitant. Cette règlementation, qui va à l'encontre de la pratique quotidienne, devrait non seulement donner du fil à retordre au corps médical, mais aussi aux pharmaciens, afin de garantir le même niveau de libre choix aux patients. Et le surcoût administratif doit finalement être payé par les patients.

La nouvelle loi propose également de modifier les catégories de médicaments. Au lieu des A, B, C, D, E usuels, seules deux catégories sont prévues: soumis ou non soumis à ordonnance. Il est également prévu que davantage de médicaments soient disponibles chez les grands distributeurs et dans les drogueries. Quelques médicaments de l'ancienne catégorie C passeront en caté-

La nouvelle loi sur les produits thérapeutiques doit viser l'amélioration de la collaboration entre les professions médicales.

gorie B. Plusieurs médicaments des catégories A et B pourront être remis directement par les pharmaciens (sans passer par un médecin) si les trois conditions suivantes sont remplies: premièrement, le patient est physiquement présent dans la pharmacie. Deuxièmement, le pharmacien note les informations nécessaires, car le risque, la responsabilité civile et la pharmacovigilance relèvent dans ce cas uniquement de lui; il les communique rapidement au médecin traitant, une étape qui fonctionne bien dans notre cabinet grâce aux télécopies, à l'internet ou au téléphone. Troisièmement, afin de garantir la sécurité des patients, seule une liste définie par des experts englobant des «médicaments et indications» utilisés pour des tableaux cliniques simples et fréquents est concernée. Le pharmacien pose préalablement l'indication exacte en tenant compte du diagnostic différentiel, de l'exclusion des contre-indications et des évolutions potentiellement dangereuses. Le groupe A contient également des médicaments à potentiel d'addiction: pouvoir se les procurer dans différentes pharmacies risque de contribuer à des remises multiples et abusives.

Dans un système "Point of Care", la remise directe de médicaments devrait être possible dans chaque cabinet médical.

Du point de vue de la santé publique, un système «Point of Care» devrait permettre à toutes les officines d'effectuer certaines prestations médicales simples et à chaque cabinet médical de remettre des médicaments, et par conséquent de rendre la propharmacie possible sur tout le territoire. Les cabinets médicaux et les pharmacies conserveront bien évidemment leurs activités principales, notamment en raison des différences fondamentales des formations respectives. L'objectif doit être de poursuivre l'amélioration de la collaboration entre les professions médicales, sans la mise en place d'un marché totalement libre ni d'une régulation inappropriée. C'est déjà ce qui se passe localement lorsque la situation est réglée au niveau cantonal. Mais à quelle condition? La confiance est assurément primordiale. Afin d'atteindre les standards de santé publique dans les projets interprofessionnels, il est plus pertinent d'associer en même temps et de manière adéquate tous les groupes professionnels concernés par la catégorisation des médicaments, plutôt que d'appliquer une stratégie sélective visant à diviser pour mieux régner. Du point de vue médical, les interventions décidées après coup par les autorités afin de limiter les dégâts sont inadaptées pour répondre à la sécurité des patients. 